\title{
Utility of Image Guidance in the Localization of Disappearing Colorectal Liver Metastases
}

\author{
Linda M. Pak, MD ${ }^{1}$, Johan Gagnière, MD, PhD ${ }^{1,2,3}$, Peter J. Allen, MD, FACS ${ }^{1,4}$, Vinod P. \\ Balachandran, MD'1 Michael I. D'Angelica, MD, FACS ${ }^{1}$, Ronald P. DeMatteo, MD, FACS ${ }^{1,5}$, \\ William R. Jarnagin, MD, FACS ${ }^{1}$, Michael I. Miga, PhD $^{6}$, Amber L. Simpson, PhD ${ }^{1}$, T. Peter \\ Kingham, MD, FACS ${ }^{1}$ \\ ${ }^{1}$ Department of Surgery, Memorial Sloan Kettering Cancer Center, 1275 York Avenue, New York, \\ NY 10065, USA
}

${ }^{2}$ Department of Digestive and Hepatobiliary Surgery, University Hospital of Clermont-Ferrand, 1 Place Lucie \& Raymond Aubrac, 63000 Clermont-Ferrand, France

${ }^{3}$ U1071 INSERM/University Clermont Auvergne, Clermont-Ferrand, France

${ }^{4}$ Department of Surgery, Duke University, 2301 Erwin Rd, Durham, NC 27110, USA

${ }^{5}$ Department of Surgery, University of Pennsylvania, 2400 Spruce Street, Philadelphia, PA 19104, USA

${ }^{6}$ Department of Biomedical Engineering, Vanderbilt University, 5901 Stevenson Center, Nashville, TN 37235, USA

\section{Abstract}

\begin{abstract}
Background-Colorectal liver metastases that demonstrate a complete radiographic response during chemotherapy are increasingly common with advances in chemotherapy regimens and are described as disappearing liver metastases (DLMs). However, these DLMs often continue to harbor residual viable tumor. If these tumors are found in the operating room with ultrasound (US), they should be treated. The intraoperative sonographic visualization of these lesions, however, can be hindered by chemotherapy-associated liver parenchyma changes. The objective of this study was to evaluate the use of an intraoperative image guidance system, Explorer (Analogic Corporation, Peabody, MA), to aid surgeons in the identification of DLMs initially undetected by US alone.
\end{abstract}

\footnotetext{
T. Peter Kingham, MD, FACS, kinghamt@mskcc.org.

Author Contributions LMP contributed to study conception and design, data acquisition, analysis, and interpretation; manuscript drafting and revision; and final approval of this version for publication, and agrees to be accountable for all aspects of this work. JG contributed to data acquisition, analysis, and interpretation; manuscript drafting and revision; and final approval of this version for publication, and agrees to be accountable for all aspects of this work. PJA, VPB, MID, RPD, and WRJ contributed to study design, data acquisition, and interpretation; manuscript drafting and revision; and final approval of this version for publication, and agree to be accountable for all aspects of this work. MIM contributed to study conception, data interpretation, manuscript revision, and final approval of this version for publication, and agrees to be accountable for all aspects of this work. ALS and TPK contributed to study conception and design, data acquisition, analysis, and interpretation; manuscript drafting and revision; and final approval of this version for publication, and agree to be accountable for all aspects of this work.

Publisher's note Springer Nature remains neutral with regard to jurisdictional claims in published maps and institutional affiliations. This study was presented as an ePoster at the 13th World Congress of the International Hepato-Pancreato-Biliary Association in Geneva, Switzerland, September 4-7, 2018.
} 
Study Design-In a single-arm prospective trial, patients with colorectal liver metastases undergoing liver resection and/or ablation with one or more DLMs during neoadjuvant chemotherapy were enrolled. Intraoperatively, DLMs were localized with conventional US. Any DLM not found by conventional US was re-evaluated with the image guidance system. The primary outcome was the proportion of sonographically occult DLMs subsequently located by image-guided US.

Results-Between April 2016 and November 2017, 25 patients with 61 DLMs were enrolled. Thirty-eight DLMs (62\%) in 14 patients (56\%) were not identified with US alone. Six (16\%) DLMs in five patients (36\%) were subsequently located with assistance of the image guidance system. The image guidance changed the intraoperative surgical plan in four of these patients.

Conclusions-Image guidance can aid surgeons in the identification of initially sonographically occult DLMs and facilitate the complete surgical clearance of all sites of liver disease.

\section{Keywords}

Image-guided surgery; Liver surgery; Navigated ultrasound; Surgical navigation; Tracked ultrasound

\section{Introduction}

Colorectal cancer is the third most common cancer in the world, with over 1 million new cases estimated annually. ${ }^{1}$ Nearly half of colorectal cancer patients will develop liver metastases, which is often the only site of distant disease. ${ }^{2,3}$ Chemotherapy, in combination with surgical resection, remains the mainstay of treatment for these patients. In some cases, patients will have liver metastases that display a radical radiographic response and can no longer be seen on serial imaging during the course of chemotherapy treatment; these are described as disappearing liver metastases (DLMs). However, this radiographic response does not always correspond with the pathologic response. ${ }^{4}$ In prior studies, only $20-64 \%$ of liver metastases that demonstrate a complete radiographic response also exhibit a complete pathologic response. ${ }^{5-7}$

This circumstance presents surgeons with a significant treatment dilemma, as DLMs often remain difficult to identify even intraoperatively with ultrasound. Radiographically occult liver lesions are a particular challenge in metastatic colorectal cancer patients, who often receive chemotherapy and can develop chemotherapy-associated alterations in the liver parenchyma, which shrink and reduce the contrast enhancement between the liver and the tumors. ${ }^{8,9}$ On ultrasound (US), this results in a hyperechoic appearance that will often obscure liver lesions that may still harbor viable cancer. ${ }^{10,11}$ Techniques to enhance conventional intraoperative US are needed to aid in the localization of these radiographically occult tumors and to guide surgeons in their surgical planning. Intraoperative image guidance has gained widespread use in other surgical fields, such as neurosurgery and orthopedic surgery, but has yet to become a standard adjunct in liver surgery. ${ }^{12-16}$

In this context, we sought to evaluate the utility of an intraoperative image guidance system, where the position and orientation of the surgeon's tool is visualized in real time with 
respect to the preoperatively acquired scan, to help guide surgeons in the identification and localization of DLMs that are initially undetected by US alone.

\section{Methods}

This is a single-arm prospective clinical trial of an FDA-approved Explorer system (Analogic Corporation, Boston, MA). Patients undergoing liver resection and/or ablation with adequate preoperative computed tomography (CT) scans were evaluated for study eligibility. Patients were included in the study if they had received neoadjuvant chemotherapy and had one or more DLMs, defined as a colorectal liver metastasis which was identified on an initial imaging scan and had subsequently become radiographically occult by the time of resection. Radiology studies were initially read by MSK radiologists, and a radiology review was performed by the primary investigator of this study (TPK). Intraoperatively, the image guidance system was used as previously described. ${ }^{17,18}$ First, the preoperative imaging data of the scan where the DLM was last visualized were downloaded from the hospital Patient Archiving and Communication System prior to surgery. Using the portal venous phase of the scan, the liver, tumors, and vasculature were manually demarcated and segmented. Next, three-dimensional (3D) models of the liver, tumor, and vasculature were constructed from the segmented imaging data. Intraoperatively, the liver was mobilized and positioned to allow sufficient exposure and access to the lesions of interest. The Aloka Alpha 7 US T probe was used to attempt initial localization of the DLMs. If all lesions were localized utilizing intra-operative anatomical landmarks, the image guidance system was not utilized, and the patient was assigned to the "Image Guidance Not Needed/Useful group" (Fig. 1). If one or more DLMs could not be located with US alone, then the system was utilized. As described previously, an optical tracking camera (Polaris Spectra, Northern Digital Inc., Waterloo, Canada) was placed at the head of the bed. ${ }^{17,19}$ An optically tracked sterile probe was used to swab four anatomic landmarks on the liver: the round ligament, the falciform ligament, and the left and right inferior borders of the liver. The system then matched the location of these four landmarks with the 3D model using a process called registration. A second optically tracked sterile rigid body was attached to the US transducer to map and overlay the US data onto the patient-specific 3D model and the imaging scan in real time. Both of these optically tracked tools, as well as the image guidance system, are available for use in open as well as minimally invasive liver surgery. ${ }^{19}$ The surgeon navigated the tracked US using the 3D model to attempt localization of the DLM with assistance of the image guidance display. If a DLM was subsequently found by US with image guidance assistance, the lesion was treated at the discretion of the surgeon (resection, ablation, or no treatment) and the patient was assigned to the "Image Guidance Useful Group.” To assess the accuracy of the system, imaging scans obtained as part of the routine standard of care postoperatively were reviewed to confirm that the targeted DLM tumor was encompassed within the area treated by resection or ablation. DLMs that were not located by US with image guidance assistance were not treated and the patient was assigned to the "Image Guidance Not Needed/Useful" group. This study was approved by the Institutional Review Board with an institutional waiver of informed consent and was HIPAA-compliant. 
Intraoperatively, the number, size, and location of the DLMs were recorded, as well as the registration time of the image guidance system, if used. Prospectively, patient age, sex, body mass index (BMI), prior chemotherapy treatment history, clinical risk score (CRS), number and location of DLMs, and date of imaging where DLMs were last visualized were collected. The primary endpoint of the study was the proportion of sonographically occult DLMs that were subsequently identified with image guidance assistance. Secondary endpoints were the proportion of patients in whom image guidance was clinically helpful (as determined by the surgeon) and the subsequent clinical trajectory of the DLMs.

Postoperatively, regardless of whether DLMs are identified or not, patients are followed with cross-sectional imaging every 4 months for the first 2 years, then every 6 months until 5 years after surgery, and then annually.

\section{Results}

\section{Patient Demographics}

Between April 2016 and October 2017, 25 patients who had received neoadjuvant chemotherapy and had one or more DLMs undergoing liver resection and/or ablation were enrolled in this study. The median duration of neoadjuvant chemotherapy treatment was 5 months (range 2-21). Overall, there were 61 DLMs, with a median of two DLMs per patient (range 1-8). The median time between the time of the last preoperative imaging scan where the DLM was radiographically visible and the time of surgery was 5.5 months (range 1.018.7 months) (Table 1). Nine patients (36\%) had both preoperative CT and MRI; no patients had preoperative MRI alone. The median size of the DLMs when they were last radiographically visible was $0.9 \mathrm{~cm}$ (range $0.2-7.0 \mathrm{~cm}$ ).

\section{Intraoperative Data}

Intraoperatively, 23 DLMs were found with US alone; 38 DLMs (62\%) in 14 patients (56\%) could not be identified with US alone. These 38 DLMs were interrogated with the image guidance system (Fig. 2). The median registration time of the image guidance system was 62 seconds (range 31-82 seconds). Of these 38 DLMs, six (16\%) DLMs in five patients (36\%) were subsequently located with assistance of the image guidance system. In three of the five patients, two had DLMs treated with microwave ablation, one with radiofrequency ablation, and one with a wedge resection. In the remaining patient with one DLM identified with image guidance assistance, no new treatment was performed as the lesion was encompassed within an area of liver parenchyma that was already included in a preplanned second-stage hepatectomy. In all cases, except for the patient with the preplanned second-stage hepatectomy, the use of the image guidance system identified one or more DLMs and subsequently changed the surgical plan intraoperatively. Figure 3 demonstrates preoperative and intraoperative images from one of the five patients with a DLM located with image guidance system assistance. A segment six lesion was noted on a preoperative CT scan 6 months before the date of surgery (Fig. 3a), which had subsequently become radiographically occult on the $\mathrm{CT}$ scan obtained immediately prior to surgery (Fig. 3b). Intraoperatively, when the DLM could not be identified with US alone, the image guidance 
system was used to guide the surgeon's examination of the region of the DLM (Fig. 3c), resulting in successful identification of the DLM (Fig. 3d).

\section{Comparison of Patient Characteristics}

A comparison of patient characteristics between those with at least one DLM identified with the image guidance system and those with DLMs that remained unidentified even with image guidance assistance is shown in Table 2. There were no significant differences in the patients' age, gender, CRS, neoadjuvant chemotherapy regimen, or number of months between the time of preoperative imaging and the time of surgery. There was a trend towards significance in the number of months of prior chemotherapy treatment between the two groups, with patients with DLM identified with image guidance assistance having had more months of neoadjuvant chemotherapy exposure.

\section{Pathologic Correlation of DLMs}

Overall, 29 DLMs were located with either US alone or US with image guidance assistance, of which 20 were resected and have pathology results available; pathology was not available for ablated tumors. All but one of the DLMs was pathologically confirmed as a colorectal liver metastasis on final pathology of the resection specimen; one DLM had a pathologic diagnosis of bile duct adenoma. Of the 19 pathologically confirmed DLMs, three demonstrated $100 \%$ treatment response and the remaining 16 DLMs showed median treatment response of $40 \%$ (range 0-98\%).

\section{Postoperative Follow-Up}

Among the three patients with DLMs identified with image guidance assistance treated with radiofrequency or microwave ablation, routine postoperative imaging showed the DLM sites encompassed within the ablation defects with no evidence of recurrence subsequently at most recent follow-up ( 6,6 , and 12 months, respectively); however, two of these patients did develop separate sites of recurrence in a different region of the liver during this follow-up period. The patient with a DLM identified with image guidance assistance that was treated with wedge resection also had routine postoperative imaging, which showed the DLM encompassed within the resection defect and no evidence of recurrence at the resection margin at time of last imaging (13 months postoperatively). For the one patient with DLM located with image guidance assistance for which treatment was deferred at the index operation and was subsequently resected during the second-stage hepatectomy, there was no evidence of recurrence at the DLM site on last imaging ( 8 months after second-stage hepatectomy); however, this patient did develop a recurrence at a separate site.

Among the nine patients with DLMs that were not identified despite image guidance assistance, two had DLMs that were already encompassed within the planned resection plan, with final surgical pathology consistent with colorectal metastases. Three patients developed liver recurrences at a DLM site, two patients developed liver recurrences separate from the DLM site, and two patients remained disease-free. 


\section{Discussion}

Advances in chemotherapy regimens have improved the outcomes for some patients with colorectal liver metastases. As increasingly more effective combinatorial regimens are being developed, more patients will have hepatic lesions that exhibit a strong or even complete radiographic response, further pressing the urgency of advances in intraoperative technologies to keep pace with this changing clinical environment. ${ }^{5,7}$ In the present study, we demonstrate the utility of the image guidance system in aiding the identification of DLMs in patients who had received a median of 5 months of preoperative chemotherapy. Of the 38 DLMs that could not be located with intraoperative US alone, the image guidance system aided in the identification of six (16\%) DLMs in five patients and informed subsequent changes in the intraoperative surgical plan in four of these patients.

DLMs pose a significant dilemma for surgeons. The standard practice is to intraoperatively interrogate all areas of disease that had been radiographically evident either before or after chemotherapy, because complete clearance of disease is the most critical factor in optimizing survival for patients with colorectal liver metastases. ${ }^{10}$ Intraoperative visualization with US becomes increasingly difficult in patients with chemotherapyassociated changes in the liver parenchyma. ${ }^{20}$ In the present study, patients with DLMs identified with image guidance had more months of prior chemotherapy exposure than those with DLMs that remained unidentified (median 7 vs 4 months). Particularly as chemotherapy exposure may be associated with an incremental degree of associated liver parenchymal alterations, these results indicate that the image guidance system was still able to provide effective assistance in identifying DLMs even in patients with extensive chemotherapy exposure and, presumably, higher grade of chemotherapy-associated parenchymal damage. ${ }^{9}, 21-23$ Among the patients who had DLMs that could not be located with US alone or with image guidance assistance, there are potentially other factors that may have limited visualization or these lesions may represent areas of true complete pathologic response. As our practice is to treat only lesions that are visualized intraoperatively, we were unable to confirm the pathology of the DLMs that were never located.

Several prior studies have addressed the limitations of using complete radiographic response as a direct measure of complete pathologic response. In a review of 38 patients with 66 DLMs, Benoist et al. found that $83 \%$ of the DLMs were either associated with residual tumor or demonstrated early recurrence at the site of the DLM. ${ }^{24}$ In a similar study of 39 patients with 118 DLMs, Auer et al. reported that $36 \%$ of DLMs harbored viable tumor or developed recurrence at the same site. ${ }^{5}$ These studies demonstrate the vast discrepancy that can occur between radiographic and true pathologic response and emphasize the need for surgeons to continue to aggressively interrogate all known sites of disease, both from before and after chemotherapy. In our practice, we do not empirically treat the localized area containing the DLM as this may result in unnecessary loss of liver volume and increase the patients' risk of postoperative liver insufficiency and other associated morbidities. In these situations, the image guidance system serves as a valuable adjunct to aid in the identification and clearance of all potential sites of residual disease. 
Other preoperative and intraoperative techniques have been proposed to facilitate the localization of DLMs. Contrast-enhanced US (CE-US) is an intraoperative technique which has been demonstrated in prior studies to aid in the identification of DLMs undetected by conventional US. In a study of 32 DLMs in 11 patients, Arita et al. reported that CEUS identified 12 additional DLMs compared to conventional US. ${ }^{25}$ While all patients in this study had received neoadjuvant chemotherapy, the duration was not specified. In comparison, in a series of 29 patients who had undergone a median of five cycles of chemotherapy with 66 DLMs, CE-US only identified one additional DLM compared to conventional US. ${ }^{26}$ Despite the addition of contrast enhancement, any intraoperative US may still be hindered by chemotherapy-associated alterations in the liver parenchyma; thus, the differences in CE-US detection between these two studies may be due to the varying duration of neoadjuvant chemotherapy exposure between these two study populations. CEUS was recently approved in the USA. It is unclear how image guidance systems and CEUS will affect each other. It is possible that this enhanced US technique will continue to face many of the same challenges that affect conventional US in the intraoperative detection of DLMs, such as the depth of the DLM, the location relative to other anatomic landmarks, and the individual surgeon's ultra-sonography skills. ${ }^{27,} 28$ In this respect, the image guidance system allows surgeons to harness their familiarity with a different imaging modality, crosssectional imaging, to facilitate their use of the intraoperative US. Improvements in preoperative imaging have also aided surgeons in the treatment algorithm of DLMs. ${ }^{29}$ In particular, the introduction of hepatobiliary phase MRI imaging, which uses a novel contrast agent, Eovist with targeted update by hepatocytes, has increased the sensitivity of preoperative radiographic detection of colorectal liver metastases. ${ }^{30,31}$ However, these remain preoperative aids and become of limited incremental value intraoperatively for locating sonographically occult lesions.

There are several limitations to the present study. First, this was not conducted in a blinded setting as the same primary surgeon performed both the initial US as well as the subsequent US with image guidance; however, this is representative of the real-world clinical situation in which the image guidance system is employed. This also addresses the effect of operator dependency in US. The surgeons whose patients were enrolled in this trial encompass a broad range of time and experience in clinical practice. While impossible to quantify the ultrasonographic skills of these surgeons, we believe that it is a reasonable assumption that they have skills that are, at minimum, at the same level with most surgeons performing liver resections. Thus, we believe that the real-world setting in which our study was conducted incorporates and reflects the potential for ultrasonographic operative variability. Second, there is no pathologic confirmation of ablated DLMs. Third, given the limited sample size of this study, we were unable to assess the individual effects of the various chemotherapy regimens on the use of the image guidance system. Lastly, the Explorer system developed by Analogic, Inc., is no longer commercially available; however, a similar image guidance system, CAS-One Liver manufactured by CAScination AG is available and has also been FDA-approved for liver surgery. The technical utility and conceptual performance of image guidance systems demonstrated in the present study will continue to translate across various system platforms. 


\section{Conclusion}

There continues to be a large gap between preoperative and intraoperative imaging. The Explorer system serves as a useful intraoperative adjunct to conventional US to aid in the identification of initially sonographically occult DLMs. By providing cross-sectional imaging correlation to intraoperative US data, image guidance systems can facilitate the complete identification and treatment of all sites of disease.

\section{References}

1. Siegel RL, Miller KD, Jemal A. Cancer statistics, 2015. CA: a cancer journal for clinicians. 2015 Jan-Feb;65(1):5-29. [PubMed: 25559415]

2. Steele G, Jr., Ravikumar TS Resection of hepatic metastases from colorectal cancer. Biologic perspective. Annals of surgery. 1989 8;210(2):127-38. [PubMed: 2667471]

3. Qiu M, Hu J, Yang D, Cosgrove DP, Xu R. Pattern of distant metastases in colorectal cancer: a SEER based study. Oncotarget. 201511 17;6(36):38658-66. [PubMed: 26484417]

4. Adam R, Wicherts DA, de Haas RJ, et al. Complete pathologic response after preoperative chemotherapy for colorectal liver metastases: myth or reality?, Journal of clinical oncology : official journal of the American Society of Clinical Oncology. 20084 1;26(10):1635-41. [PubMed: 18375892]

5. Auer RC, White RR, Kemeny NE, et al. Predictors of a true complete response among disappearing liver metastases from colorectal cancer after chemotherapy. Cancer. 20103 15;116(6):1502-9. [PubMed: 20120032]

6. Ferrero A, Langella S, Russolillo N, Vigano L, Lo Tesoriere R, Capussotti L. Intraoperative detection of disappearing colorectal liver metastases as a predictor of residual disease. Journal of gastrointestinal surgery : official journal of the Society for Surgery of the Alimentary Tract. 2012 4;16(4):806-14. [PubMed: 22258869]

7. van Vledder MG, de Jong MC, Pawlik TM, Schulick RD, Diaz LA, Choti MA. Disappearing colorectal liver metastases after chemo-therapy: should we be concerned? Journal of gastrointestinal surgery : official journal of the Society for Surgery of the Alimentary Tract. 2010 11;14(11):1691700. [PubMed: 20839072]

8. Peppercorn PD, Reznek RH, Wilson P, Slevin ML, Gupta RK. Demonstration of hepatic steatosis by computerized tomography in patients receiving 5-fluorouracil-based therapy for advanced colorectal cancer. British journal of cancer. 1998 6;77(11):2008-11. [PubMed: 9667683]

9. Zorzi D, Laurent A, Pawlik TM, Lauwers GY, Vauthey JN, Abdalla EK. Chemotherapy-associated hepatotoxicity and surgery for colorectal liver metastases. The British journal of surgery. 2007 3;94(3):274-86. [PubMed: 17315288]

10. Carpenter S, Fong Y. Management of disappearing colorectal hepatic metastases. Advances in surgery. 2010;44:269-79. [PubMed: 20919526]

11. Valls C, Iannacconne R, Alba E, et al. Fat in the liver: diagnosis and characterization. European radiology. 2006 10;16(10):2292-308. [PubMed: 16477402]

12. Jolesz FA, Kikinis R, Talos IF. Neuronavigation in interventional MR imaging. Frameless stereotaxy. Neuroimaging clinics of North America. 2001 11;11(4):685-93, ix. [PubMed: 11995423]

13. Benardete EA, Leonard MA, Weiner HL. Comparison of frameless stereotactic systems: accuracy, precision, and applications. Neurosurgery 2001 12;49(6):1409-15; discussion 15-6. [PubMed: 11846941]

14. Nasser R, Drazin D, Nakhla J, et al. Resection of spinal column tumors utilizing image-guided navigation: a multicenter analysis. Neurosurg Focus. 2016 8;41(2):E15.

15. Zumwalt TJ, Goel A. Immunotherapy of Metastatic Colorectal Cancer: Prevailing Challenges and New Perspectives. Current colorectal cancer reports. 20156 1;11(3):125-40. [PubMed: 26441489] 
16. Pishnamaz M, Wilkmann C, Na HS, et al. Electromagnetic Real Time Navigation in the Region of the Posterior Pelvic Ring: An Experimental In-Vitro Feasibility Study and Comparison of Image Guided Techniques. PloS one. 2016;11(2):e0148199. [PubMed: 26863310]

17. Kingham TP, Scherer MA, Neese BW, Clements LW, Stefansic JD, Jarnagin WR. Image-guided liver surgery: intraoperative projection of computed tomography images utilizing tracked ultrasound. HPB : the official journal of the International Hepato Pancreato Biliary Association. 2012 9;14(9):594-603. [PubMed: 22882196]

18. Kingham TP, Pak LM, Simpson AL, et al. 3D image guidance assisted identification of colorectal cancer liver metastases not seen on intraoperative ultrasound: results from a prospective trial. HPB (Oxford). 2018 3;20(3):260-67. [PubMed: 28935452]

19. Kingham TP, Jayaraman S, Clements LW, Scherer MA, Stefansic JD, Jarnagin WR. Evolution of image-guided liver surgery: transition from open to laparoscopic procedures. Journal of gastrointestinal surgery : official journal of the Society for Surgery of the Alimentary Tract. 2013 7;17(7):1274-82. [PubMed: 23645420]

20. van Vledder MG, Torbenson MS, Pawlik TM, et al. The effect of steatosis on echogenicity of colorectal liver metastases on intraoperative ultrasonography, Archives of surgery (Chicago, Ill : 1960). 2010 7;145(7):661-7.

21. Karoui M, Penna C, Amin-Hashem M, et al. Influence of preoperative chemotherapy on the risk of major hepatectomy for colorectal liver metastases. Annals of surgery. 2006 1;243(1):1-7. [PubMed: 16371728]

22. Fernandez FG, Ritter J, Goodwin JW, Linehan DC, Hawkins WG, Strasberg SM. Effect of steatohepatitis associated with irinotecan or oxaliplatin pretreatment on resectability of hepatic colorectal metastases. Journal of the American College of Surgeons. 2005 6;200(6):845-53. [PubMed: 15922194]

23. Kneuertz PJ, Maithel SK, Staley CA, Kooby DA. Chemotherapy-associated liver injury: impact on surgical management of colorectal cancer liver metastases. Annals of surgical oncology. 2011 1;18(1):181-90. [PubMed: 20645011]

24. Benoist S, Brouquet A, Penna C, et al. Complete response of colorectal liver metastases after chemotherapy: does it mean cure?, Journal of clinical oncology : official journal of the American Society of Clinical Oncology. 20068 20;24(24):3939-45. [PubMed: 16921046]

25. Arita J, Ono Y, Takahashi M, Inoue Y, Takahashi Y, Saiura A. Usefulness of contrast-enhanced intraoperative ultrasound in identifying disappearing liver metastases from colorectal carcinoma after chemotherapy. Annals of surgical oncology. 2014 6;21 Suppl 3:S390-7. [PubMed: 24570378]

26. Sturesson C, Nilsson J, Lindell G, Andersson RG, Keussen I. Disappearing liver metastases from colorectal cancer: impact of modern imaging modalities. HPB (Oxford). 2015 11;17(11): 983-7. [PubMed: 26252426]

27. Torzilli G, Botea F, Donadon M, et al. Criteria for the selective use of contrast-enhanced intraoperative ultrasound during surgery for colorectal liver metastases. HPB : the official journal of the International Hepato Pancreato Biliary Association. 2014 11;16(11):994-1001. [PubMed: 24830573]

28. Zendel A, Lahat E, Dreznik Y, Zakai BB, Eshkenazy R, Ariche A. "Vanishing liver metastases"-A real challenge for liver surgeons. Hepatobiliary surgery and nutrition. 2014 10;3(5):295-302. [PubMed: 25392841]

29. Fowler KJ, Linehan DC, Menias CO. Colorectal liver metastases: state of the art imaging. Annals of surgical oncology. 2013 4;20(4):1185-93. [PubMed: 23115006]

30. Niekel MC, Bipat S, Stoker J. Diagnostic imaging of colorectal liver metastases with CT, MR imaging, FDG PET, and/or FDG PET/CT: a meta-analysis of prospective studies including patients who have not previously undergone treatment. Radiology. 2010 12;257(3):674-84. [PubMed: 20829538]

31. Park MJ, Hong N, Han K, et al. Use of Imaging to Predict Complete Response of Colorectal Liver Metastases after Chemotherapy: MR Imaging versus CT Imaging. Radiology. 2017 8;284(2):42331. [PubMed: 28327002] 


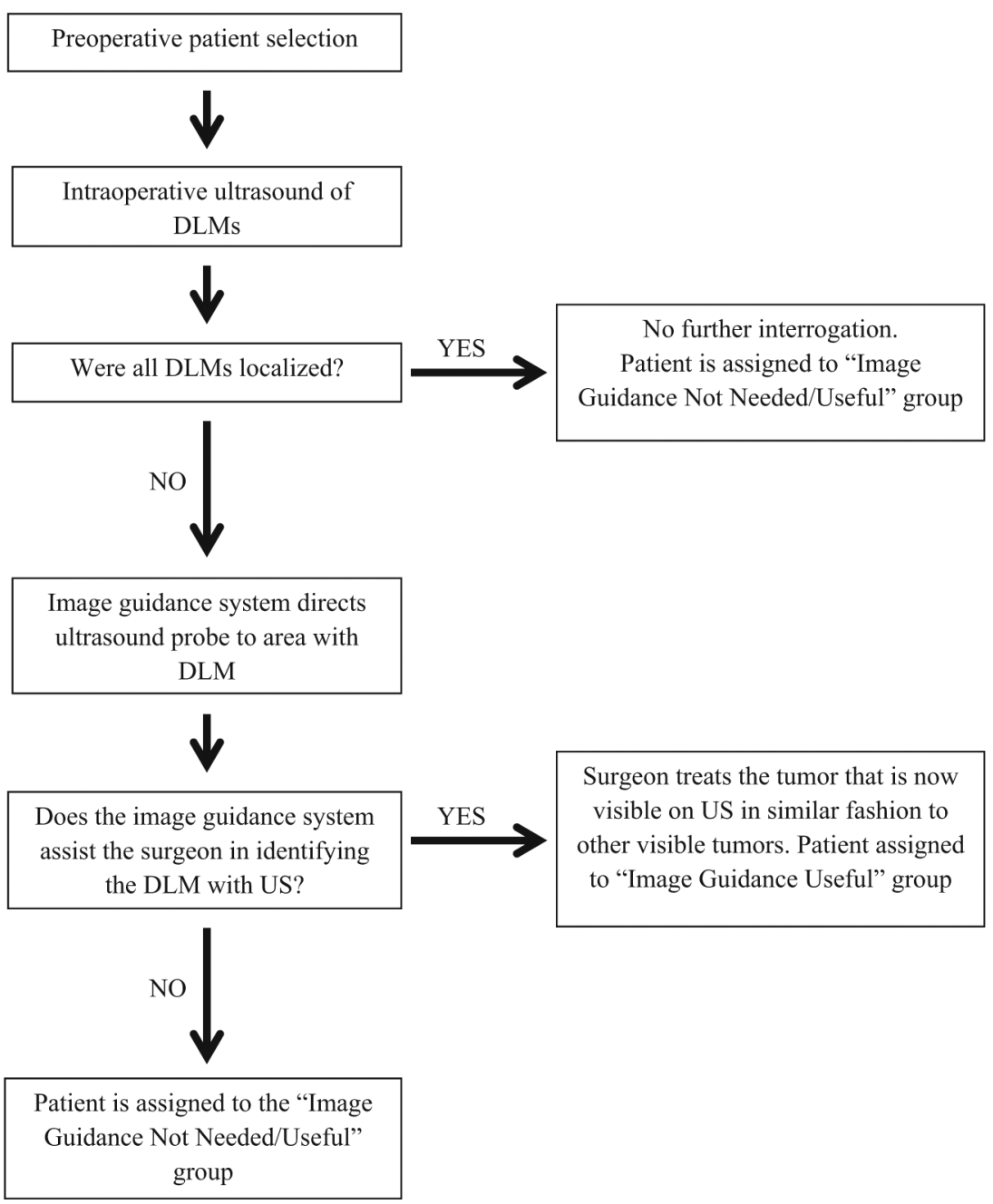

Fig. 1.

Study workflow diagram 


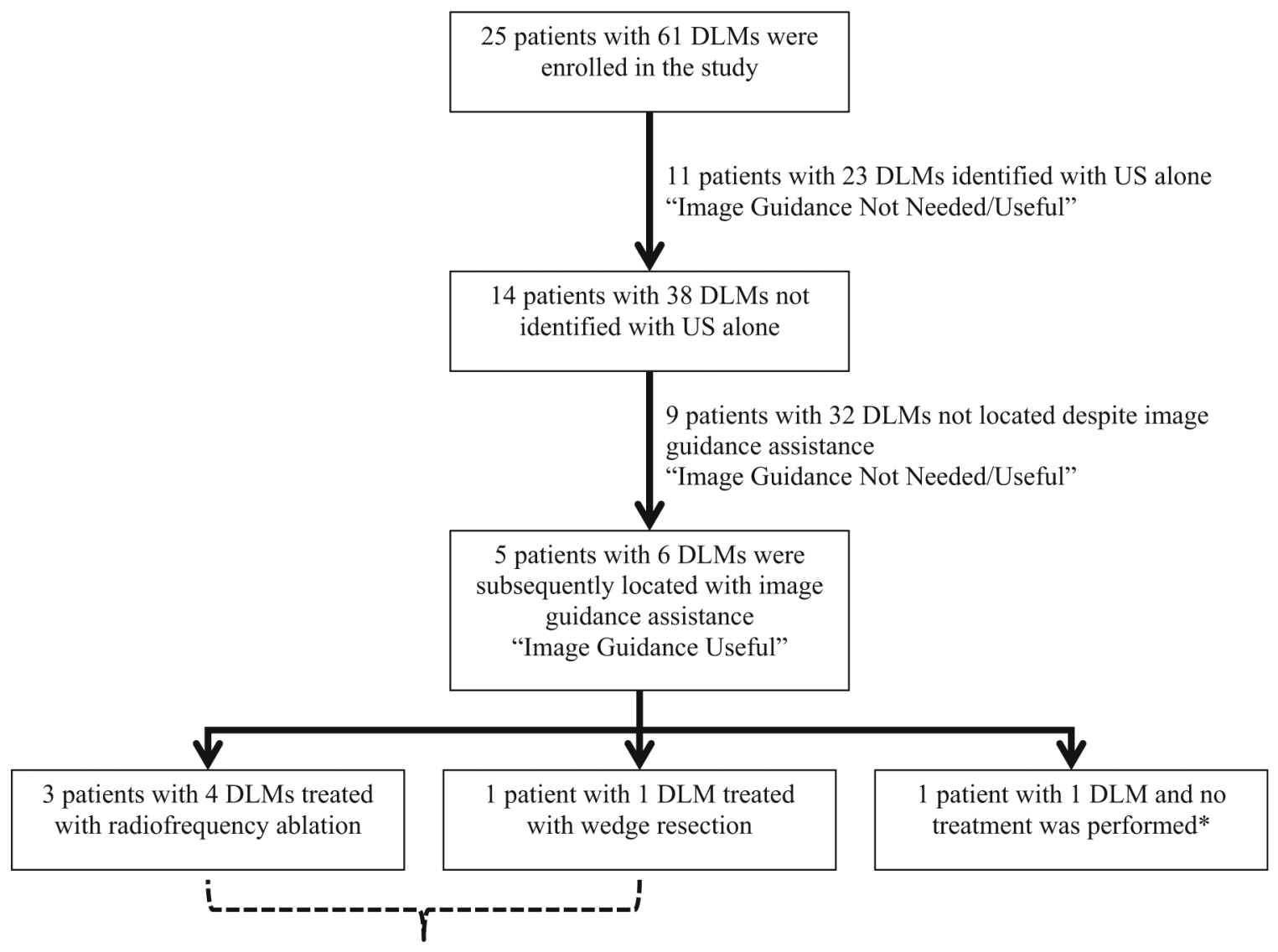

Explorer assistance changed intraoperative surgical management

Fig. 2. Intraoperative image guidance use and treatment details

*Location of DLM was encompassed within resection area of pre-planned second-stage hepatectomy 

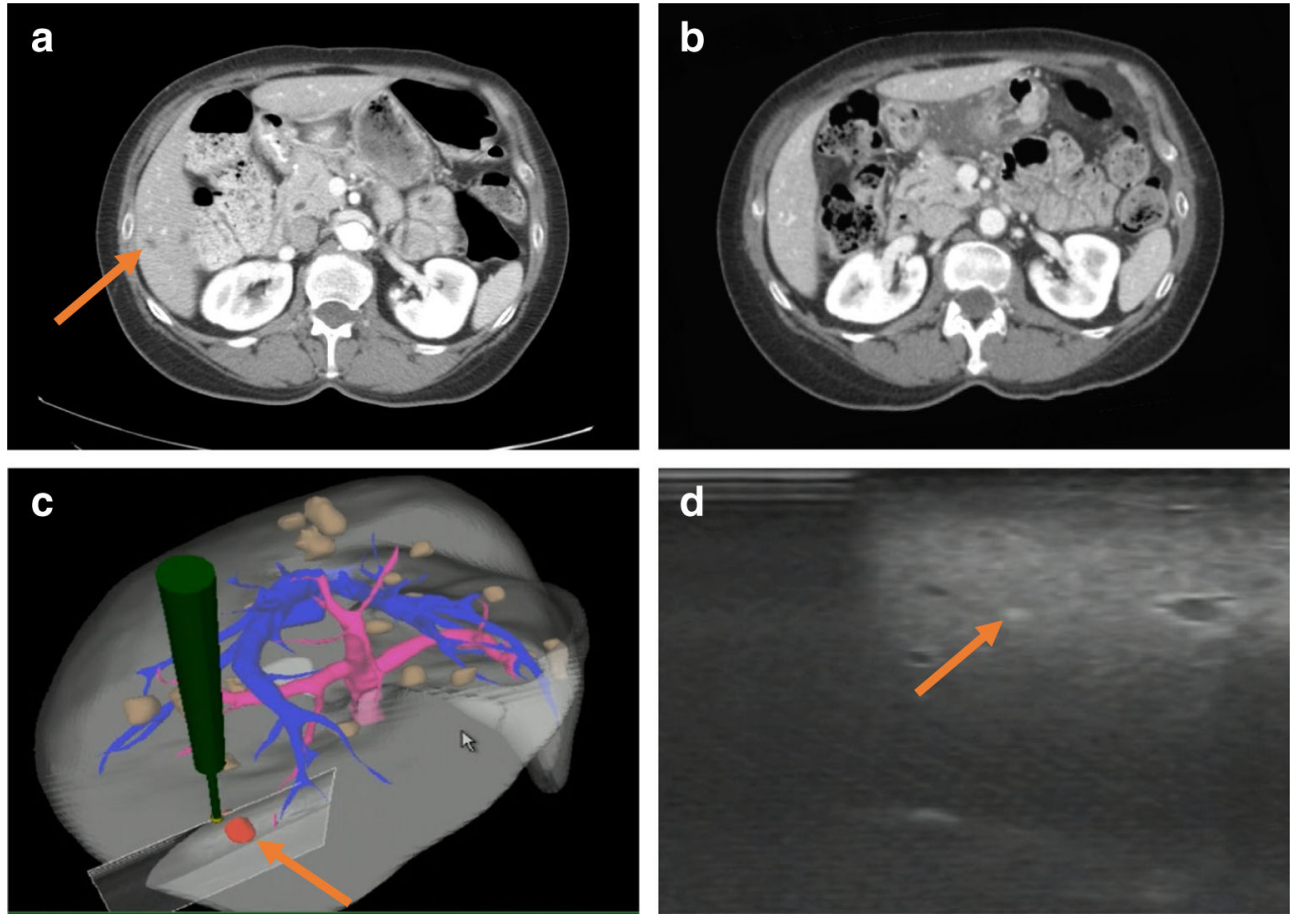

Fig. 3.

Image guidance system in use. a Last preoperative CT scan where DLM (orange arrow) was radiographically visible, 6 months before date of surgery. $\mathbf{b}$ Corresponding section of immediately-preoperative CT scan where DLM has become radiographically occult. c Intraoperative mapping of area of intraoperative US exam to 3D model of the liver with DLM (orange arrow). d Successful identification of DLM (orange arrow) on intraoperative US with image guidance 


\section{롤 \\ 골}
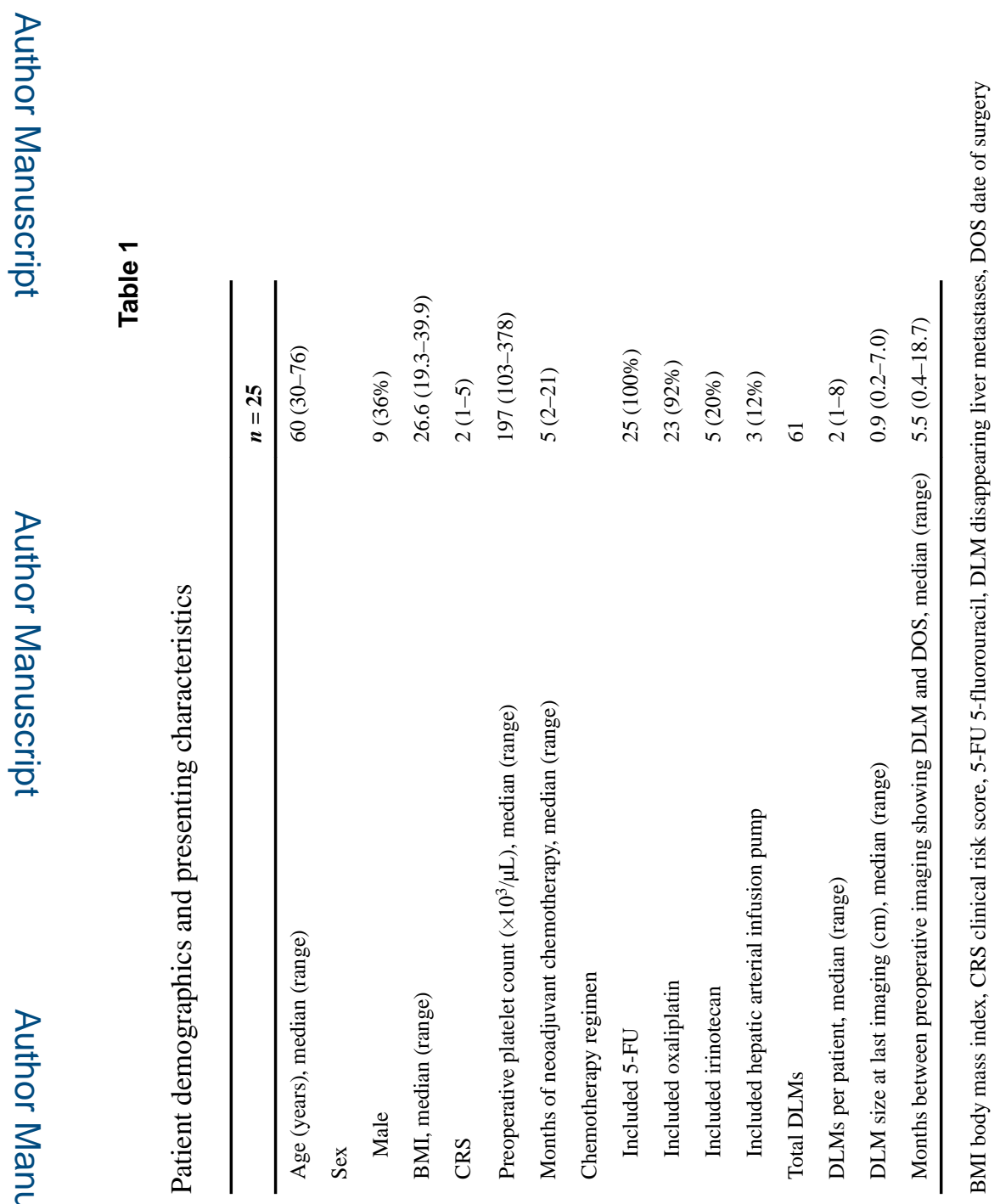

J Gastrointest Surg. Author manuscript; available in PMC 2019 August 31. 
Pak et al.

Page 14

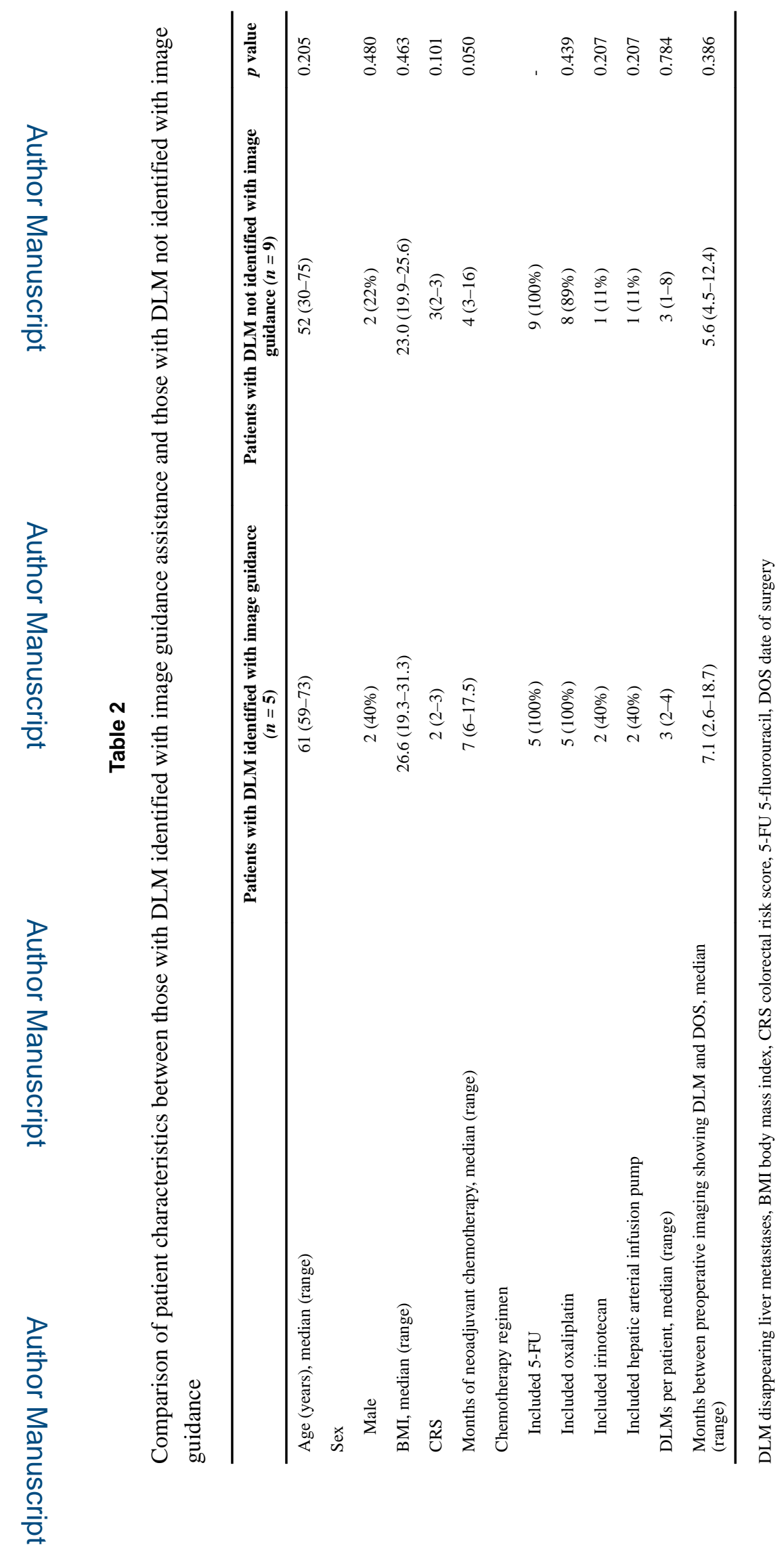

J Gastrointest Surg. Author manuscript; available in PMC 2019 August 31. 